\title{
ESTABILIDADE DE EMULSÕES FRENTE A DIFERENTES TÉCNICAS DE HOMOGENEIZAÇÃO E RESFRIAMENTO.
}

\section{STABILITY OF EMULSIONS FRONT OF DIFFERENT TECHNIQUES OF HOMOGENIZATION AND COOLING.}

\author{
'BARZOTTO, I. L. M.; ${ }^{2}$ OLIVEIRA, S. M. M.; ${ }^{3}$ TAVARES, B.; ${ }^{4}$ DALLABRIDA, S. \\ 1,2 Docentes do Curso de Farmácia da UNIOESTE - PR \\ Email: simone_meneoli@yahoo.com.br \\ ${ }^{3,4}$ Acadêmicas do Curso de Farmácia da UNIOESTE - PR \\ REC: 06/09 AC: 07/09
}

\begin{abstract}
RESUMO:
Na farmácia de manipulação, as emulsões são os veículos ideais para diversas aplicações cosméticas e farmacêuticas, pois reúnem qualidades estéticas e funcionais, como a solubilização de componentes hidro e lipofílicos. As emulsões precisam demonstrar estabilidade física sob as mais variadas condições, como as variações de temperatura e o estresse induzido pelas vibrações durante o transporte. Devem, também, manter a consistência adequada para conseguir boa sensação sobre a pele, espalhar-se e liberar ingredientes ativos. $\mathrm{O}$ estudo de estabilidade de produtos cosméticos fornece informações sobre o comportamento do produto, indicando seu grau de estabilidade em determinado intervalo de tempo, frente a condições ambientais a que possa ser submetido, desde a fabricação até o término do prazo de validade. O objetivo deste trabalho foi verificar a estabilidade e os caracteres organolépticos de emulsões, após o preparo das mesmas, utilizando diferentes técnicas de homogeneização e resfriamento. Para a realização dos testes de estabilidade foram utilizadas duas formulações, uma emulsão aniônica, o Creme Lanette ${ }^{\circledR}$ e uma emulsão não iônica, o Creme Polawax ${ }^{\circledR}$. Após a pesagem das matérias-primas, aquecimento e mistura das fases, a homogeneização foi realizada de quatro maneiras diferentes, quanto à temperatura e velocidade de agitação, até atingir a temperatura de $40^{\circ} \mathrm{C}$. Depois de submetidos a testes de estabilidade, não foram verificadas diferenças significativas nas características dos cremes estudados, mediante as técnicas de homogeneização e resfriamento aplicados.
\end{abstract}

Palavras chave: creme não iônico, creme aniônico, farmácia de manipulação

\begin{abstract}
:
In manipulation pharmacy, emulsions are the ideal vehicle for various cosmetic and pharmaceutical applications, because they bring aesthetic and functional qualities, such as solubilization of hydro and lipophilic components. Emulsions must demonstrate physical stability under different conditions, such as variations in temperature and stress induced by vibration during transport. They should also maintain the proper consistency to get a good feel on the skin, spread and release the active ingredients. The stability study of cosmetic products provides information on the performance of the product, indicating their degree of stability in a given time interval, compared to the environmental conditions that can be submitted from manufacturing until the end of life. The purpose of this study was to verify the stability and organoleptic characteristics of two emulsions, anionic and non-ionic, after preparing using different techniques of homogenization and cooling. To perform the stability tests were used two formulations, an anionic emulsion, Lanette Cream and a non-ionic emulsion, the cream polawax. After weighing the raw materials, heating and mixing of the phases, homogenization was performed in four different ways, as the temperature and agitation speed, reaching a temperature of 40 C. After being tested for stability, there were
\end{abstract}


no significant differences in the characteristics of the creams studied by the techniques of homogenization and cooling applied.

Key words: nonionic cream, anionic cream, manipulation pharmacy.

\section{INTRODUÇÃO}

Na farmácia de manipulação, as emulsões são os veículos ideais para diversas aplicações cosméticas e farmacêuticas, pois reúnem qualidades estéticas e funcionais, como a solubilização de componentes hidro e lipofílicos (HIR, 1997). A palavra emulsão aplica-se, de modo geral, a todas as preparações de aspecto leitoso, com características de sistema disperso constituído por duas fases líquidas imiscíveis (FONSECA e PRISTA, 2000), resultante da dispersão de um líquido no seio de outro, no qual é miscível, à custa de um agente emulsificante (HIR, 1997).

Entre os diferentes tipos de emulsões existentes, as que contêm emulsionante não-iônico são amplamente empregadas em formulações cosméticas devido às suas características e propriedades, como compatibilidade com o pH cutâneo, estabilidade frente a diferentes valores de $\mathrm{pH}$ e a possibilidade de incorporação de diferentes substâncias ativas (MILAN, 2007).

O método geral ou direto de preparação consiste em adicionar gradualmente a fase interna, mantendo agitação constante, à fase externa, tendo em solução a totalidade dos agentes tensoativos, acrescentando, por fim, o líquido restante. Todas as substâncias incluídas na fórmula serão previamente dissolvidas em óleo ou em água, conforme sua solubilidade. A fase oleosa e a fase aquosa estarão na mesma temperatura. Para proceder a emulsificação é necessário agitar. Pelo método direto se junta a fase interna à fase externa, fundem-se os matérias graxos a temperatura de $70^{\circ} \mathrm{C}$ e aquece-se a fase aquosa a mesma temperatura. Pelo método indireto, quando uma das fases ultrapassa 74\%, haverá inversão de fase (BARATA, 2002).

As emulsões usadas nas formulações de cosméticos precisam demonstrar estabilidade física sob as mais variadas condições, como as variações de temperatura e o estresse induzido pelas vibrações durante o transporte. Devem, também, manter a consistência adequada para conseguir boa sensação sobre a pele, espalhar-se e liberar ingredientes ativos (TADROS, 2007). Emulsão estável é aquela que conserva as devidas proporções entre seus constituintes e mantêm a superfície interfásica, mesmo após estar exposta a tensões decorrentes de fatores como temperatura, agitação e aceleração da gravidade (SILVA e SOARES, 1996). No entanto, as emulsões são preparações cosméticas instáveis, termodinamicamente falando. Com o tempo, apresentam sinais progressivos de instabilidade, podendo ocorrer separação de fases (LEONARDI, 2004).

O estudo de estabilidade de produtos cosméticos fornece indicações sobre o comportamento do produto, indicando seu grau de estabilidade em determinado intervalo de tempo, frente a condições ambientais a que possa ser submetido, desde a fabricação até o término da validade (ANVISA, 2004).

"Os estudos de estabilidade podem ser classificados de acordo com sua duração, 
em estudos de curto e longo prazo. Os de curto prazo (envelhecimento acelerado) têm por finalidade prever a vida útil do produto, quando estiver sob as condições ambientais de armazenamento de uso (LEONARDI, 2004)".

Dentre os tipos de estabilidade, existe a física, onde são mantidas as propriedades físicas originais, inclusive aparência, textura, uniformidade, cor e odor; e a química, onde cada substancia ativa mantêm sua integridade química (LEONARDI, 2004). A emulsão tem esse tipo de estabilidade quando não apresenta quebras macroscópicas, Ela é dependente da temperatura, do tempo, da densidade e viscosidade. Na estabilidade química é necessário prever instabilidade entre os princípios ativos (ZANIN, 2001; HIR, 1997). Alterações de $\mathrm{pH}$, reações de oxido-redução e/ou de hidrólise podem levar a alterações da atividade das substancias ativas, das características organolépticas e físicas das emulsões (ANVISA, 2004). A instabilidade física pode ser observada quando ocorrem fenômenos de coalescência, cremeação, e floculação. (SANCTIS, 1999).

As propriedades de uma emulsão dependem de vários fatores, tais como componentes da preparação, temperatura de emulsificação, velocidade de resfriamento, intensidade de agitação e ordem de adição de matérias-primas (SANCTIS, 1999, ANVISA, 2004).

Conforme a origem, as alterações podem também ser classificadas como intrínsecas (fatores inerentes à formulação) e/ou extrínsecas (fatores externos), segundo Anvisa (2004). Os fatores extrínsecos são referentes a fatores externos, aos quais a emulsão esteja exposta, tais como tempo, temperatura, umidade, microrganismos e vibração. Fatores Intrínsecos são fatores relacionados à própria formulação e a interações de seus ingredientes, entre si ou com o material de acondicionamento (ANVISA, 2004).

\section{OBJETIVO}

Verificar a estabilidade e os caracteres organolépticos de duas emulsões aniônica e não-iônica, após o preparo das mesmas utilizando diferentes técnicas de homogeneização e resfriamento.

\section{MATERIAL E MÉTODOS}

Os ensaios foram realizados no laboratório de Farmacotécnica e Cosmetologia da Universidade Estadual do Oeste do Paraná. Para a realização dos testes de estabilidade foram utilizadas duas formulações, uma emulsão aniônica, o Creme Lanette ${ }^{\circledR}$ e uma emulsão não iônica, o Creme Polawax®.

Na formulação do Creme Lanette $®$ foi utilizado na fase $\mathrm{A}$ (oleosa): Cera lanette $\mathrm{N}$ $7 \%$, vaselina líquida $5 \%$. Fase B (aquosa): Glicerina $5 \%$, nipagim $0,1 \%$, água destilada q.s.p. $100 \mathrm{~g}$ do produto.

No Creme Polawax ${ }^{\circledR}$ foi utilizado na fase A (oleosa): Polawax NF 14\%, óleo mineral 5\%. Fase B (aquosa): Glicerina 5\%, Nipagim 0,1\%, EDTA/BHT 0,01, Água destilada q.s.p. $100 \mathrm{~g}$ do produto (SAMPAIO, 1999).

As emulsões foram então preparadas pesando as matérias primas em balança analítica, transferidas para béqueres e aquecidas separadamente (fase A e fase B) 
em banho-maria, até atingirem temperatura de $75^{\circ} \mathrm{C}$. Em seguida, a fase aquosa foi vertida sobre a fase oleosa.

A partir desta etapa, a homogeneização foi realizada de quatro maneiras diferentes, quanto à temperatura e velocidade de agitação, até atingir a temperatura de 40ㄷ. As diferentes formas foram: MaA: Agitação manual em temperatura ambiente; MaG: Agitação manual em banho de gelo; MeA: Agitação mecânica em temperatura ambiente; MeG: Agitação mecânica em banho de gelo.

\subsection{Amostragem}

Foram retiradas amostras de $30 \mathrm{~g}$ cada uma e acondicionadas em temperaturas diferentes, em triplicata, para avaliar a estabilidade das emulsões e seus parâmetros físico-químicos, totalizando 24 amostras.

Todas as amostras foram submetidas a 3 leituras para a determinação das características físico-químicas.

\subsection{Parâmetros físico-químicos avaliados}

As características físico-químicas estudadas nas amostras foram aspecto/ aparência, $\mathrm{pH}$, densidade, separação/sedimentação.

\subsection{Aspecto/aparência desejada}

Para avaliar o aspecto das emulsões, primeiramente foram definidas quais as qualidades desejáveis e as indesejáveis do produto (Quadro 1).

QUADRO 1. CARACTERÍSTICAS ORGANOLÉPTICAS PASSÍVEIS DE SEREM OBSERVADAS NAS EMULSÕES.

\begin{tabular}{lcc}
\hline Qualidades desejáveis & Defeitos aceitáveis & Defeitos sérios \\
\hline Homogeneidade & - & Heterogêneo \\
Brilho & Mate & Opaco \\
Macio & - & Fibroso \\
Fino & - & Grosso \\
Opacidade & Translúcido & Opalescente \\
& Perolado & - \\
- & - & Bolhas de ar
\end{tabular}

Fonte: SAMPAIO, 1999

\section{$3.4 \mathrm{pH}$}

Para determinação do $\mathrm{pH}$, as amostras foram diluídas em água destilada numa 
concentração de 1:10.

\subsection{Densidade}

A densidade foi determinada por meio de um picnômetro metálico com capacidade para $10 \mathrm{~g}$.

\subsection{Separação e sedimentação (centrifugação)}

As amostras foram centrifugadas em temperatura, tempo e velocidade padronizados em 2500 rpm por 30 minutos em temperatura ambiente. Em seguida avaliou-se visualmente a amostra.

\subsection{Testes de estabilidade}

Para a verificação da estabilidade dos cremes preparados, foram realizados os seguintes testes físicos: a. Estufa a 45 C durante uma semana; b. Congelador a -5C durante uma semana; c. Exposição ao sol em temperatura ambiente durante uma semana; d. Ciclos de temperatura (estufa a $45^{\circ} \mathrm{C}$, exposição ao sol em temperatura ambiente, congelador a $-^{\circ} \mathrm{O}$ ) durante $24 \mathrm{~h}$ cada (SAMPAIO, 1999).

\section{RESULTADOS E DISCUSSÃO}

Os resultados obtidos foram organizados em tabelas. A tabela 1 mostra as alterações apresentadas pelos cremes preparados com as quatro diferentes técnicas de homogeneização e resfriamento e submetidos aos testes de estabilidade. Os produtos foram enumerados de um a oito, sendo que os mesmos cremes enumerados na tabela 1 são representados pelos mesmos números na tabela 2 .

TABELA 1 TESTES DE ESTABILIDADE DOS CREMES LANETTE E POLAWAX PREPARADOS DE DIFERENTES MANEIRAS.

\begin{tabular}{|c|c|c|c|c|c|c|c|}
\hline № & Fórmula & $\begin{array}{c}\text { Técnica de } \\
\text { homog. e } \\
\text { resfriamento }\end{array}$ & Estufa a $40^{\circ} \mathrm{C}$ & Congelador $-5^{\circ} \mathrm{C}$ & $\begin{array}{l}\text { Sol / Temp. } \\
\text { ambiente }\end{array}$ & $\begin{array}{l}\text { Ciclos de } \\
\text { Temp. }\end{array}$ & Centrifugação \\
\hline 1 & Lanette & $\mathrm{MaA}$ & $\mathrm{N}$ & $\mathrm{N}$ & $\mathrm{N}$ & $\mathrm{N}$ & Cremeação \\
\hline 2 & Lanette & $\mathrm{MeA}$ & $N$ & $N$ & $N$ & $N$ & Cremeação \\
\hline 3 & Lanette & $\mathrm{MaG}$ & $N$ & $N$ & $N$ & $N$ & Cremeação \\
\hline 4 & Lanette & MeG & $N$ & $N$ & $N$ & $N$ & Cremeação \\
\hline 5 & Polawax & $\mathrm{MaA}$ & $\begin{array}{l}\text { levemente } \\
\text { ressecado }\end{array}$ & $N$ & $N$ & $N$ & $\mathrm{~N}$ \\
\hline 6 & Polawax & $\mathrm{MeA}$ & $\begin{array}{l}\text { levemente } \\
\text { ressecado }\end{array}$ & $N$ & $N$ & $N$ & $N$ \\
\hline 7 & Polawax & $\mathrm{MaG}$ & $\begin{array}{l}\text { levemente } \\
\text { ressecado }\end{array}$ & $N$ & $N$ & $N$ & $N$ \\
\hline 8 & Polawax & $\mathrm{MeG}$ & $\begin{array}{l}\text { levemente } \\
\text { ressecado }\end{array}$ & $N$ & $\mathrm{~N}$ & $\mathrm{~N}$ & $\mathrm{~N}$ \\
\hline
\end{tabular}

N: Normal; MaA: Agitação manual à temperatura ambiente; MaG: Agitação manual em banho de gelo; MeA: Agitação mecânica em temperatura ambiente; MeG: Agitação mecânica em banho de gelo. 
Foi perceptível a sensibilidade do creme Lanette frente ao teste de centrifugação, pois foi observada cremeação do mesmo. No entanto, o mesmo ocorreu com todas as formulações lanette, o que indica que o problema encontra-se no desequilíbrio entre os componentes da fórmula, e não na técnica de homogeneização e resfriamento, pois não houve diferenciação de resultado entre as técnicas.

Quanto ao creme Polawax foi observada instabilidade em relação a altas temperaturas, tornando-se levemente ressecado quando submetido ao calor da estufa. No entanto, esta instabilidade também ocorreu com todas as formulações polawax, indicando que as diferentes técnicas de homogeneização e resfriamento não influenciaram no produto.

Quanto aos demais testes, ambos os cremes mantiveram-se estáveis.

As características organolépticas e de espalhamento observadas em cada creme são apresentadas na tabela 2 .

TABELA 2. CARACTERÍSTICAS ORGANOLÉPTICAS E DE ESPALHAMENTO APRESENTADAS PELAS EMULSÕES ESTUDADAS.

\begin{tabular}{|c|c|c|c|c|c|c|c|c|c|}
\hline № & Aspecto & Brilho & Textura & Cor & Odor & $\begin{array}{l}\text { Espalha- } \\
\text { mento }\end{array}$ & Cobertura & $\begin{array}{c}\text { Branquea- } \\
\text { mento }\end{array}$ & Penetração \\
\hline 1 & Homog & Opaco & Macio & Branco & Caract & Flexível & Alta & Fraco & Lenta \\
\hline 2 & Heterog & Opaco & Macio & Branco & Caract & Flexível & Alta & Fraco & Lenta \\
\hline 3 & Heterog & Opaco & Macio & Branco & Caract & Flexível & Alta & Fraco & Lenta \\
\hline 4 & Heterog & Opaco & Macio & Branco & Caract & Flexível & Alta & Fraco & Lenta \\
\hline 5 & Homog & Brilhoso & Macio & $\begin{array}{c}\text { Branco } \\
\text { amarelado }\end{array}$ & Caract & $\begin{array}{l}\text { Flexível/ } \\
\text { grudento }\end{array}$ & Alta & Fraco & Rápida \\
\hline 6 & Homog & Brilhoso & Macio & $\begin{array}{c}\text { Branco } \\
\text { amarelado }\end{array}$ & Caract & $\begin{array}{l}\text { Flexível/ } \\
\text { grudento }\end{array}$ & Alta & Fraco & Rápida \\
\hline 7 & Homog & Brilhoso & Macio & $\begin{array}{c}\text { Branco } \\
\text { amarelado }\end{array}$ & Caract & $\begin{array}{l}\text { Flexível/ } \\
\text { grudento }\end{array}$ & Alta & Fraco & Rápida \\
\hline 8 & Homog & Brilhoso & Macio & $\begin{array}{c}\text { Branco } \\
\text { amarelado }\end{array}$ & Caract & $\begin{array}{l}\text { Flexível/ } \\
\text { grudento }\end{array}$ & Alta & Fraco & Rápida \\
\hline
\end{tabular}

Homog= Homogêneo; Heterog. $=$ Heterogêneo; Caract. $=$ Característico.

As diferentes técnicas de homogeneização e resfriamento não interferiram significativamente nas características organolépticas do produto manipulado, sendo que as emulsões mantiveram os mesmos caracteres.

\section{CONCLUSÃO}

A estabilidade e os caracteres organolépticos das duas emulsões analisadas não apresentaram diferença significativa entre os mesmos, depois de submetidos às diferentes técnicas de homogeneização e resfriamento.

Conclui-se, portanto, que pode ser utilizada qualquer uma das quatro técnicas apresentadas neste trabalho para a homogeneização e resfriamento destes cremes, sem que a mesma provoque qualquer alteração nas características das formulações. 


\section{REFERÊNCIAS}

HIR A. L. Noções de Farmácia Galênica. $6^{\underline{a}}$ ed. Ed. Organização Andrei, São Paulo 1997. pg 176-184.

AMARAL, M. P. H.; VILELA, M. A. P. Controle de Qualidade na Farmácia de Manipulação. Ed. UFRJ. 1를. Juiz de Fora, MG. 2002.

ANVISA, Guia de estabilidade de produtos cosméticos. v.1, Maio/2004.

BARATA, E. A. F. A Cosmetologia - Princípios Básicos. Ed. Tecnopress. 2ª Ed. São Paulo, 2002. p. 55-56.

FLORENCE, A. T.; ATTWOOD, D.; ZULEIKA, R. Princípios físico-químicos em farmácia. Ed USP, São Paulo, 2003. p. 360-362.

FONSECA, A.; PRISTA, L.N. Manual de Terapêutica Dermatológica e Cosmetologia. Ed. Roca. 1를. São Paulo, 2000. p. 24.

LEONARDI, G. R. Cosmetologia Aplicada. São Paulo: Medfarma, 2004. p 51,9698.

MILAN, A. L. K.; MILÃO, D.; ARIGONY, A. S., CORTE, T. W. F.; Estudo da hidratação da pele por emulsões cosméticas para xerose e sua estabilidade por reologia. Brazilian Journal of Pharmaceutical Sciences. vol. 43, n. 4, out./dez., 2007. P. 649-657.

SAMPAIO, A.C. Curso avançado de cremes e loções cremosas. Consulcom, São Paulo, 1999.

SANCTIS, D. S. Emulsões para Uso Externo. Racine, São Paulo, ano IX, n.53, p.5362. Nov/Dez 1999.

SANCTIS, D. S. Emulsões: Aplicações em cremes e loções cosméticas. Artigo técnico, Oxigeno, 2004.

SILVA, E. C.; SOARES, I.C. Tecnologia de Emulsões. Cosmetics \& Toiletries, Edição em português. v. 8, n. 4, p. 37-38,40,44-45. Set/Out. 1996.

SOUZA, F. L. C. Desenvolvimento de bases emulsionadas de silicone e água e avaliação comparativa com bases emulsionadas de óleo e água para uso externo de uso mais comum em manipulação. Dissertação de mestrado - UFRJ, Rio de Janeiro, 2007.

TADROS, T.; NESTOR, J.; TAELMAN, M. C.; SMITS, R. Emulsões: correlação da estabilidade física de longo prazo com medições reológicas de curto prazo. Cosmetics \& Toiletries, Brasil, v. 19, n. 2, Mar-Abr/2007. p. 60-65.

ZANIN, S. M. W. Parâmetros físicos no estudo da estabilidade das emulsões. Revista Visão Acadêmica, Curitiba, v. 2, n. 2, p. 47-58, Jul.-Dez./2001. 